\title{
GPM MICROWAVE IMAGER KEY TECHNOLOGIES, PERFORMANCE AND CALIBRATION RESULTS
}

David Newell, Don Figgins, David Draper, Barry Berdanier, Michael Kubitschek, David Holshouser and Adam Sexton Ball Aerospace \& Technologies Corp. Boulder, Colorado USA

Sergey Krimchansky

NASA Goddard Space Flight Center

Frank Wentz, and Thomas Meissner

Remote Sensing Systems, Santa Rosa California, USA

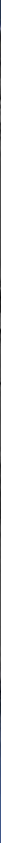




\section{GMI is Operating On-Orbit and Providing Outstanding Performance}

- GMI was launched February $28^{\text {th }}$ from Tanegashima Japan.

- The GMI main reflector was successfully deployed with the deployment time similar to ground operations

- The spin mechanism launch restraints were all successfully released

- GMI was turned on 4 days after launch and has operated continuously with no anomalies

- The data from the first 3 months of operations has been analyzed and shows that the instrument is meeting all requirements with excellent performance for the key calibration uncertainty and NEDT requirements

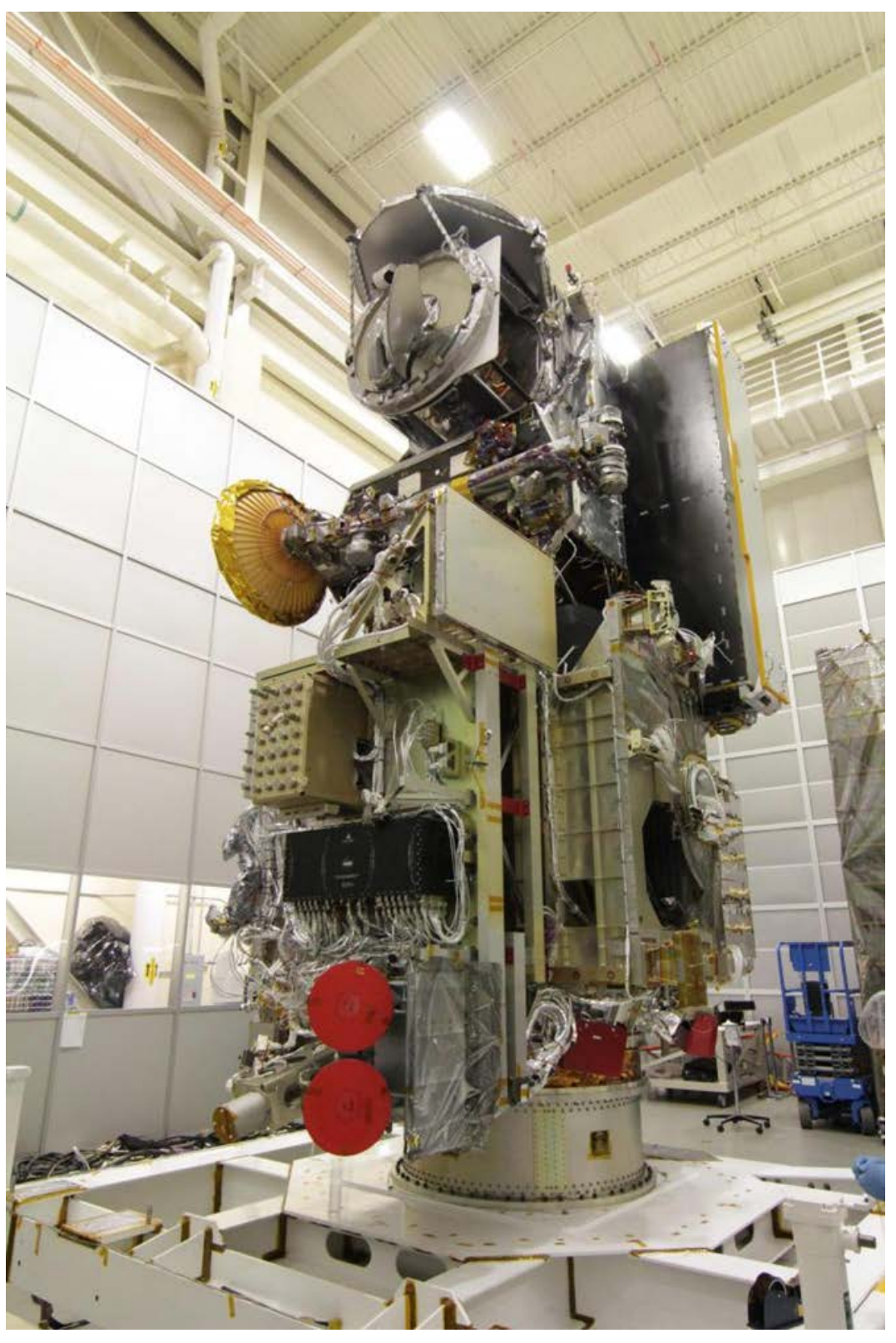




\section{GPM Core Observatory Launched Feb 28, 2014}
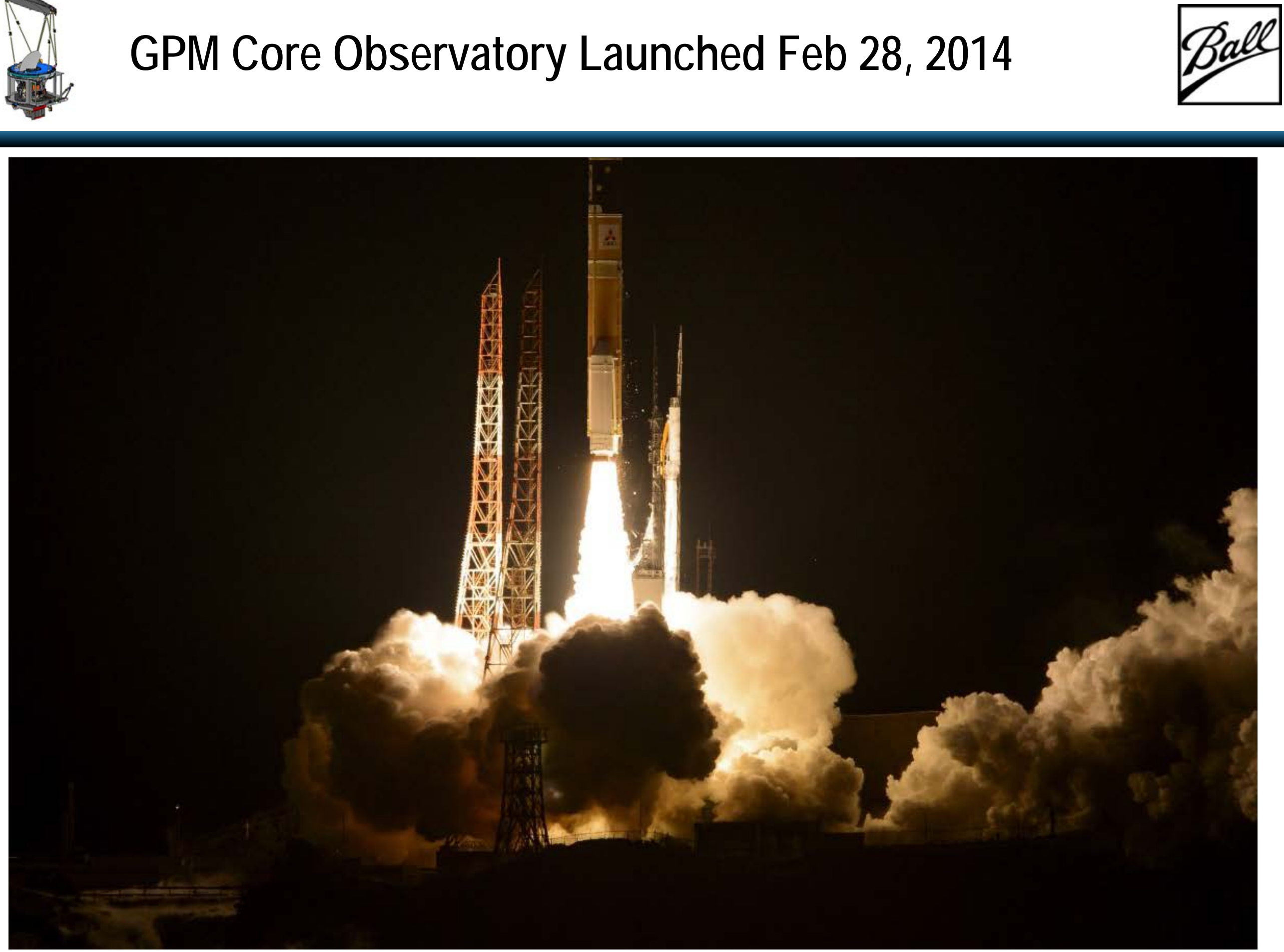


\section{GPM Mission Overview}

- The Global Precipitation Measurement (GPM) Mission is an international effort managed by the National Aeronautics and Space Administration (NASA) to improve climate, weather, and hydrometeorological predictions through more accurate and more frequent precipitation measurements

- The GPM Microwave Imager (GMI) is currently operating on the Core GPM Spacecraft and being used to make calibrated, radiometric measurements from space at multiple microwave frequencies and polarizations

- The Core GPM Spacecraft is flying at $407 \mathrm{~km}$ in a circular 65 degree inclination orbit

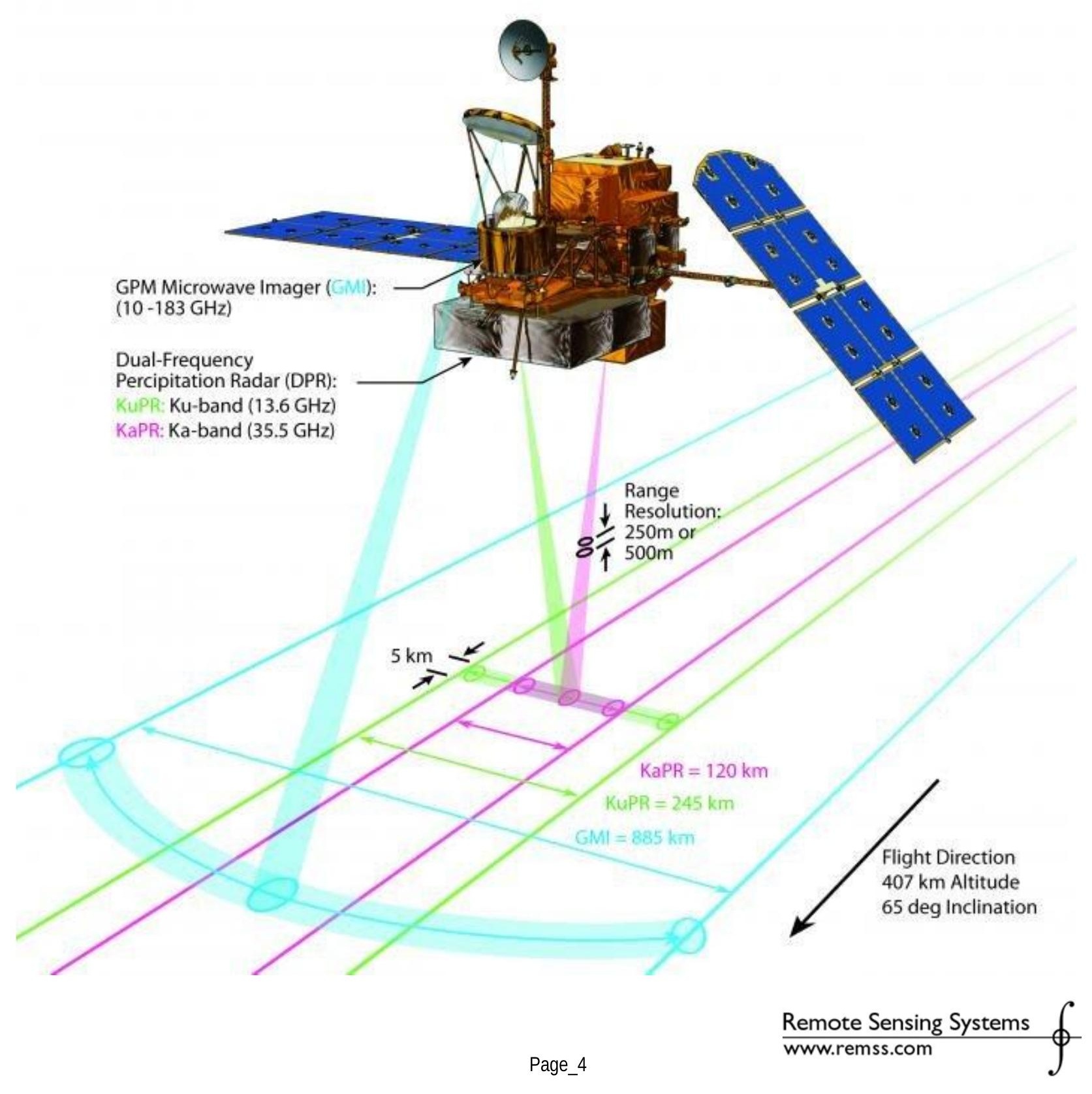




\section{GPM Observation of Hurricane Arthur}

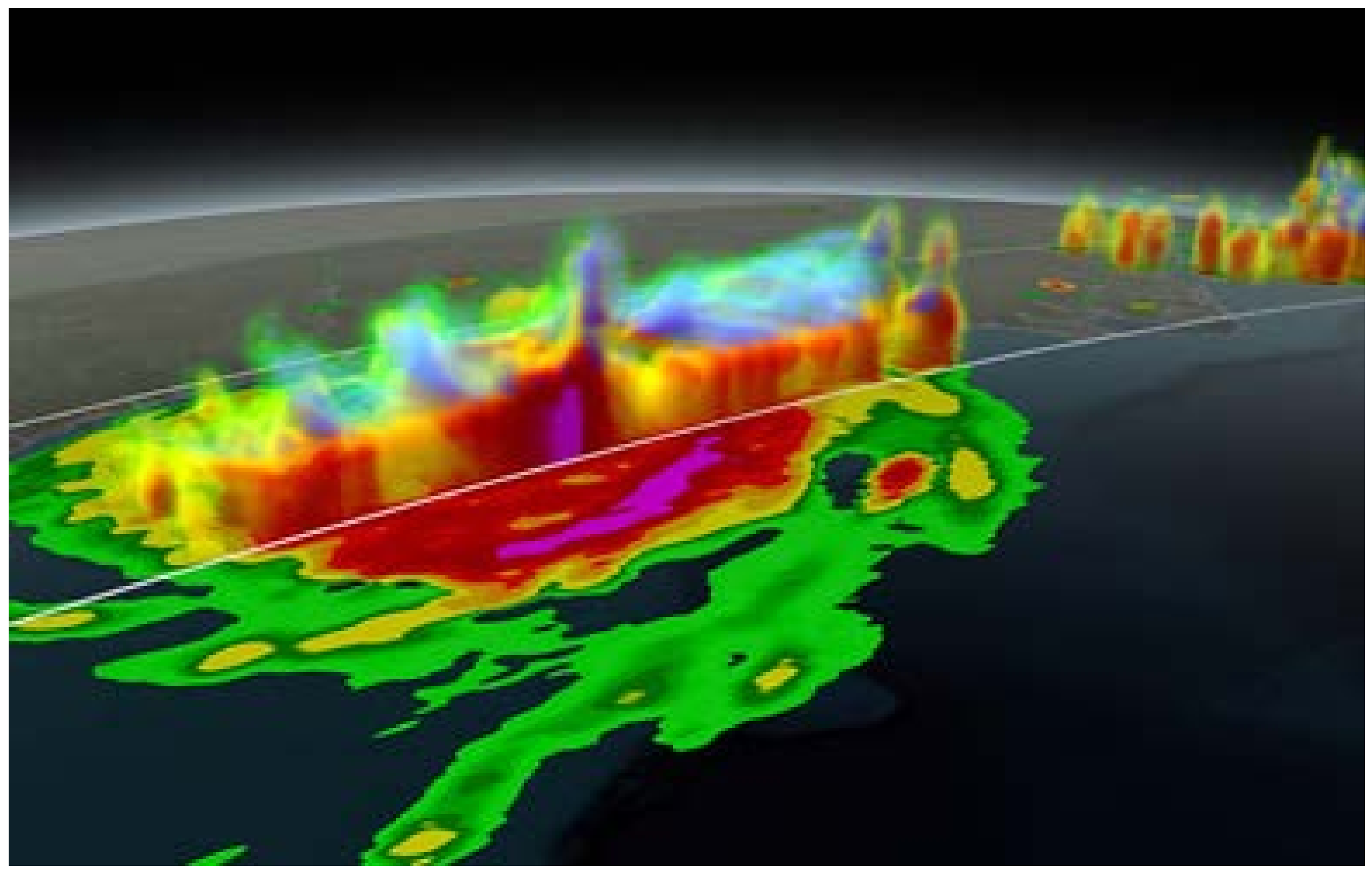




\section{GMI Instrument Overview}

- The GMI design is a total power passive radiometer with through-the-feed hot and cold calibration

- The instrument is conically scanned with rotation about the vertical axis

- The radiometer has channels at $10.65 \mathrm{GHz}(\mathrm{V} / \mathrm{H})$, 18.7(V/H) GHz, 23.8 (V) GHz, 36.64 (V/H) GHz, 89 (V/H) $\mathrm{GHz}, 166(\mathrm{~V} / \mathrm{H}) \mathrm{GHz}$ and 183.31 (V) GHz

- The measured mass is $168 \mathrm{~kg}$ and the measured onorbit power is power is 146 watts.

- The allocated launch envelope requires the antenna to be stowed on the side of the instrument for launch

- The GMI instrument included a number of design features to ensure stability and calibration accuracy which have been proven effective on-orbit

- Noise diodes in addition to hot and cold calibration targets for $10-36 \mathrm{GHz}$

- Enclosed hot target to prevent sun loading

- Sunshade to thermally isolate the receivers to minimize orbital temperature variations due to sun loading

- Large cold sky reflector to minimize earth sidelobe contribution

- Custom vapor deposited aluminum main reflector coating with high reflectivity up to $183 \mathrm{GHz}$

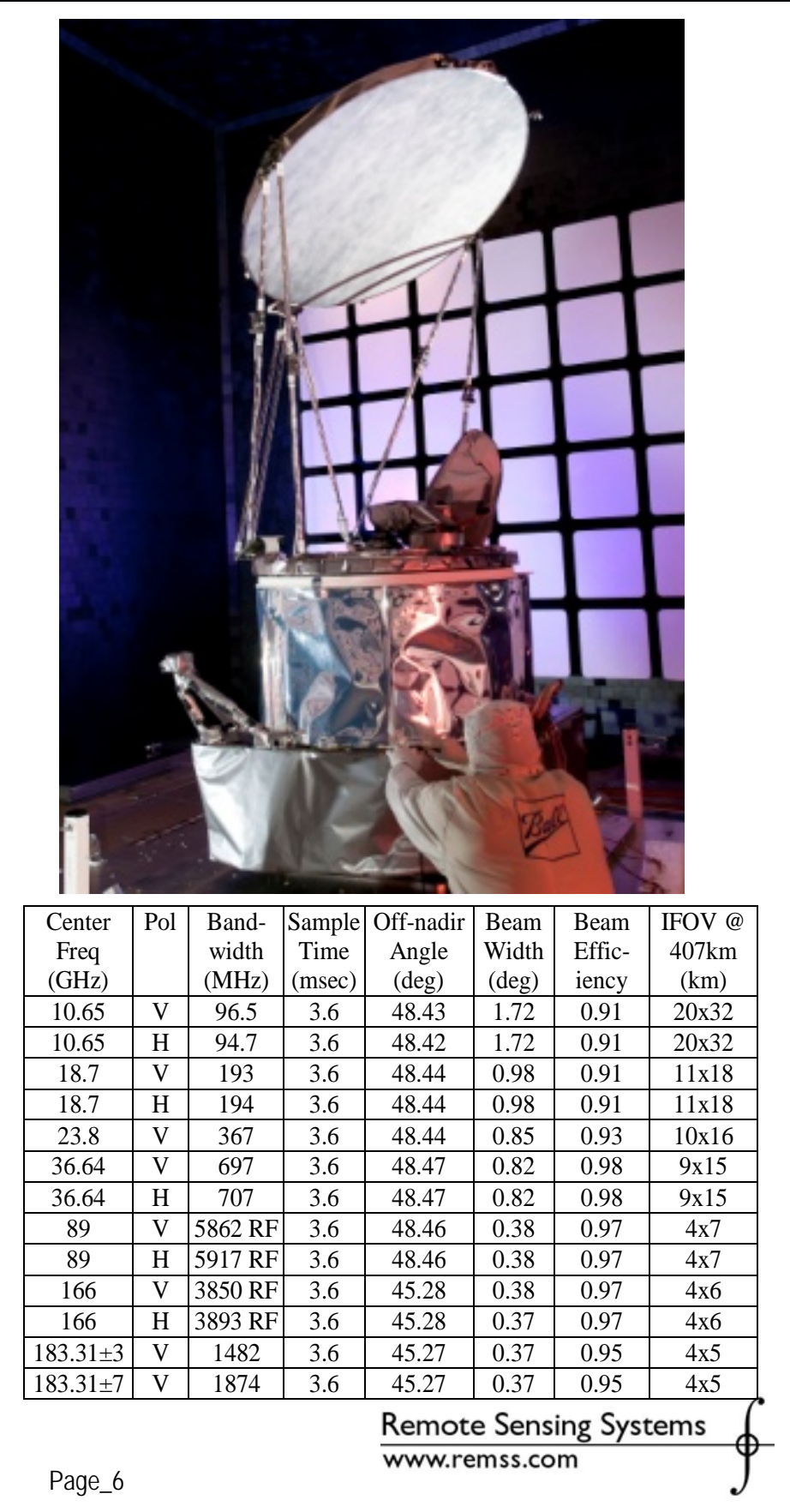




\section{GMI Measured Performance Meets or Exceeds All Requirements}

- The performance of the GMI instrument has been updated using the on-orbit performance

- The instrument performance against key requirements is shown in the adjacent tables

- In the key requirements of NEDT and calibration accuracy the GMI has significant margin to the required performance

- The receivers have operated with excellent thermal gain stability, varying by channel from $0.004 \mathrm{~dB} / \mathrm{K}$ and $0.06 \mathrm{~dB} / \mathrm{K}$

- This compares to the instrument thermal design of $0.1 \mathrm{~K} /$ minute maximum thermal variation, the contribution to NEDT from thermal gain drifts is negligible.

- In addition, the receivers operate with very little $1 / f$ noise, which contributes only a few percent to the NEDT

- The non-linearity has been verified onorbit using the noise diodes and found to match the ground measurements closely

- The non-linearity will be monitored throughout the mission

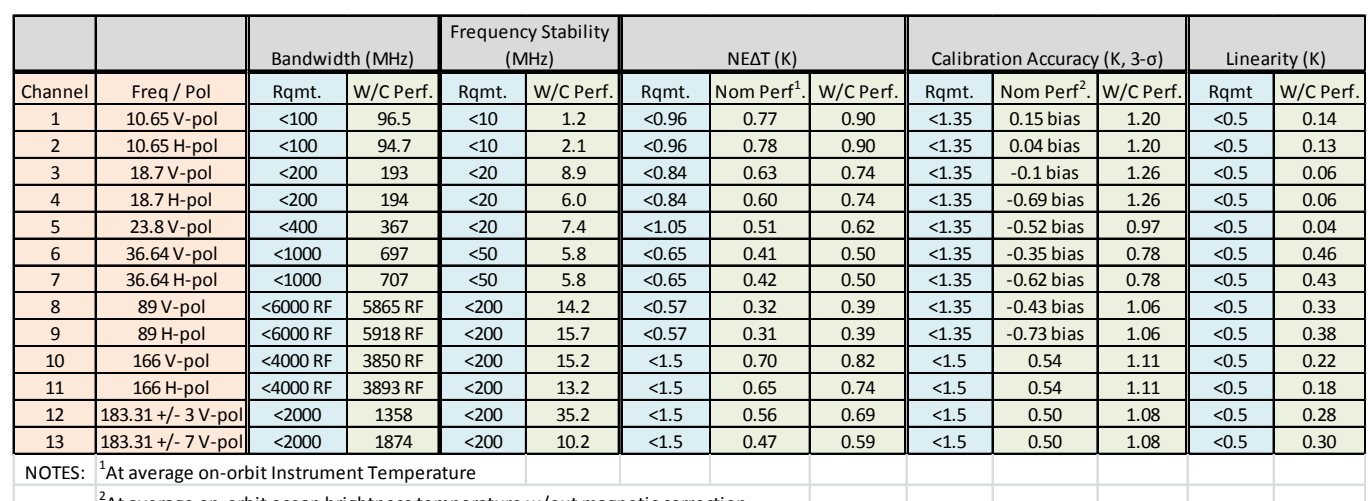

${ }^{2} A t$ average on-orbit ocean brightness temperature w/out magnetic correction
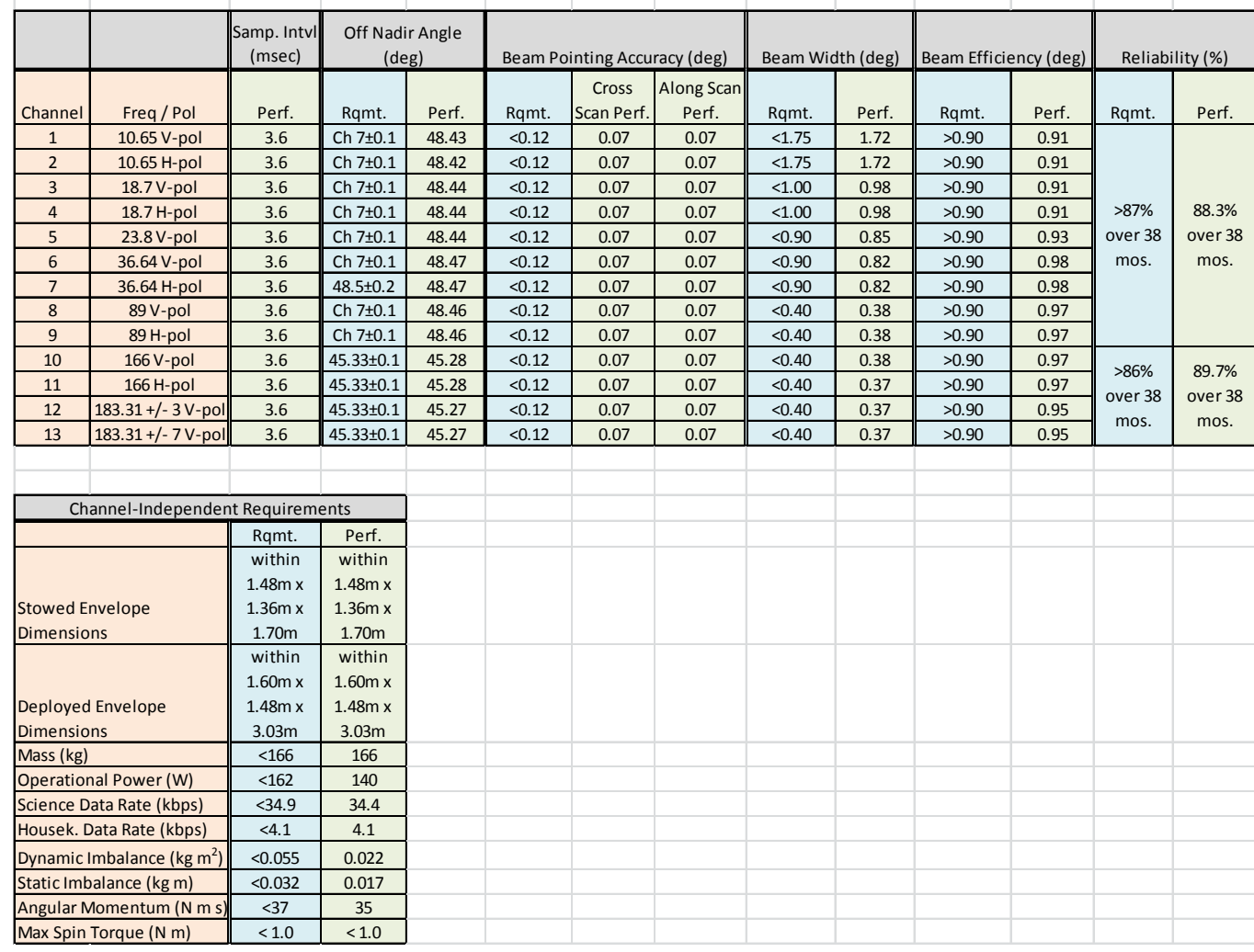


\section{Noise Diode Performance}

- The GMI provides noise diodes on the 10.65 through $36.64 \mathrm{GHz}$ channels that provide a method to perform backup calibration if the hot load or cold sky views experience transient anomalies.

- The noise diodes also provide a method to trend non-linearity on orbit.

- The stability of the noise diode output power can be monitored using the radiometer calibrated using the hot and cold sky targets

- The graphs show the GMI noise diode deviation from the characterization as a function of time for the first 3 months of operations.

- Spikes correspond to moon or RFI intrusion into the cold sky view. Long spikes are spacecraft maneuvers.

- The $10.65 \mathrm{~V}$-pol noise diode and the $36 \mathrm{~V}$-pol noise diode have experienced some on-orbit drift.

- There is a shift in noise diode excess temperature behavior around 3/22/2014 for the $10 \mathrm{~V}$ and $36 \mathrm{~V}$ noise diodes.

- The GMI noise diodes will continue to be monitored for stability.
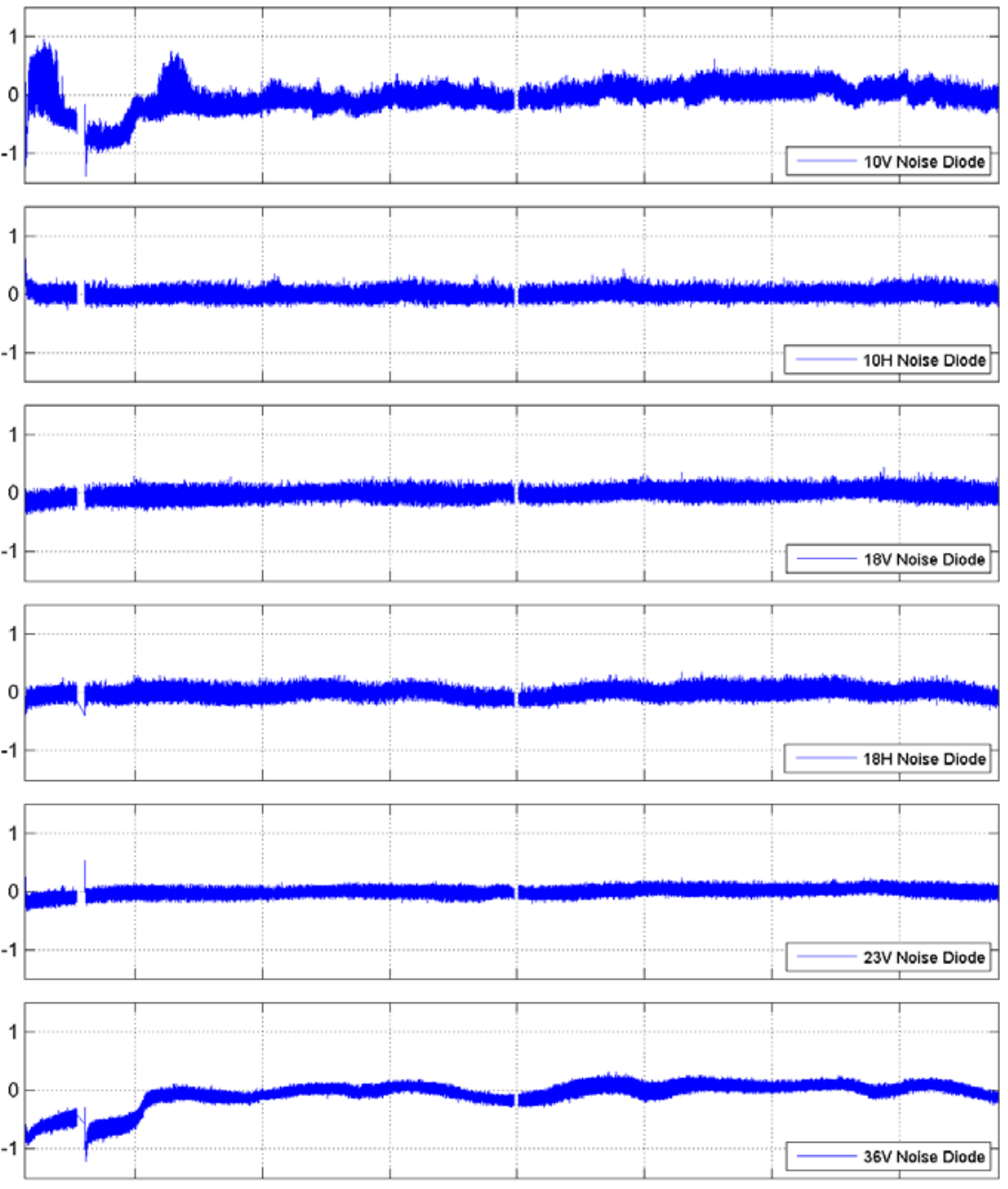

\begin{tabular}{|c|c|c|c|}
\hline $\begin{array}{c}\text { Center } \\
\text { Freq } \\
(\mathrm{GHz})\end{array}$ & Pol & $\begin{array}{c}\text { N. Diode } \\
\text { Drift, } \\
\text { mo (K) }\end{array}$ & $\begin{array}{c}\text { N.Diode } \\
\text { Orbit Var } \\
\pm \mathrm{pk}(\mathrm{K})\end{array}$ \\
\hline 10.65 & $\mathrm{~V}$ & 0.7 & \pm 0.3 \\
\hline 10.65 & $\mathrm{H}$ & 0.13 & \pm 0.3 \\
\hline 18.7 & $\mathrm{~V}$ & 0.11 & \pm 0.25 \\
\hline 18.7 & $\mathrm{H}$ & 0.13 & \pm 0.25 \\
\hline 23.8 & $\mathrm{~V}$ & 0.14 & \pm 0.25 \\
\hline 36.64 & $\mathrm{~V}$ & 0.8 & \pm 0.35 \\
\hline 36.64 & $\mathrm{H}$ & 0.14 & \pm 0.2 \\
\hline
\end{tabular}

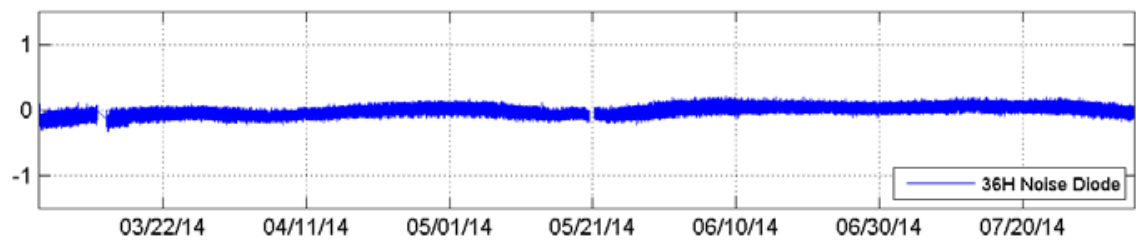




\section{Hot Target Performance is Stable Over All Solar Beta Angles}

- The figure shows the $\mathbf{1 5}$ thermistors located on the hot load over a $\mathbf{4 5}$ day beta angle cycle

- The temperatures are well behaved and do not show any spikes from solar intrusions

- This confirms that the design features added to the warm load are working

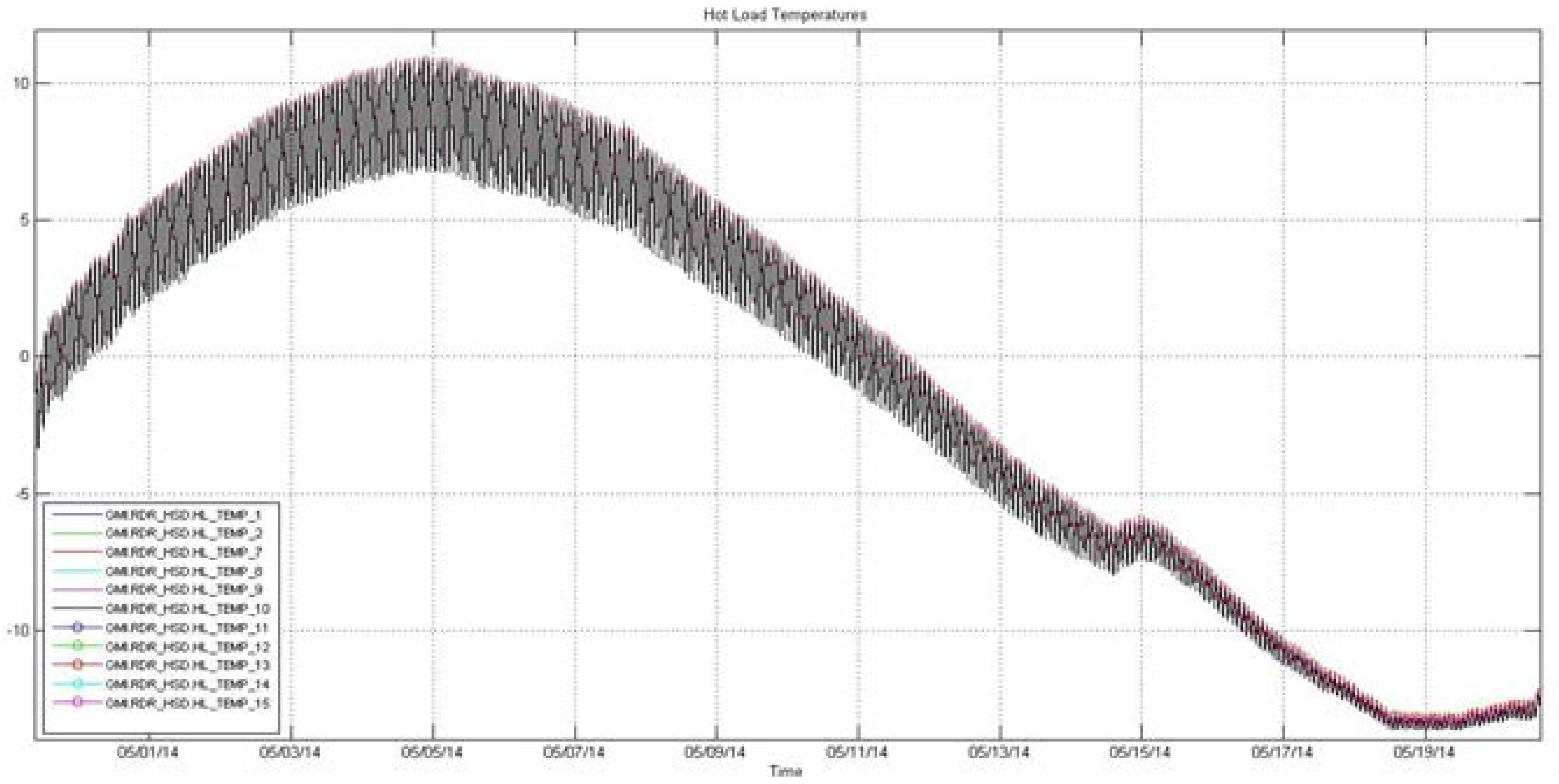




\section{Calibration Uncertainty}

- The basic tool for the GMI post-launch calibration method is to compare antenna temperatures (TA) that are measured from the radiometer with those computed from the radiative transfer model (RTM) developed by Remote Sensing Systems (RSS).

- The difference between measured and RTM computed TA is used to remove the variability of the environmental scene.

- WindSat and AMSR-2 measurements that are collocated with GMI and temporally located within one hour are used for comparison

- The collocated WindSat and AMSR-2 measurements are used to determine ocean surface wind speed, columnar water vapor and columnar liquid cloud water

* The WindSat and AMSR-2 ocean products have been carefully validated by RSS to remove WindSat/AMSR-2 instrument biases using ground truth data

- For SST the Reynolds OI product is used. The wind direction is taken from NCEP GDAS

- These parameters are input into the RTM and a TA calculated for GMI

- The calculated GMI TA is compared to the measured TA

- The first 90 days of data is shown on the following slides

- Along scan biases have been determined and removed

- Orbital biases due to a small magnetic susceptablity in some changes has been determined and removed 


\section{GMI Minus RTM TA Difference Versus Orbit Number and Orbit Position: H-POL No Magnetic Susceptability Correction}

- A 0.5 Kelvin bias that is orbit position dependent can be seen at $10.7 \mathrm{GHz}$

- This has been determined to be caused by the magnetic field of the earth and a correction algorithm is planned to be implemented in the flight algorithm in September
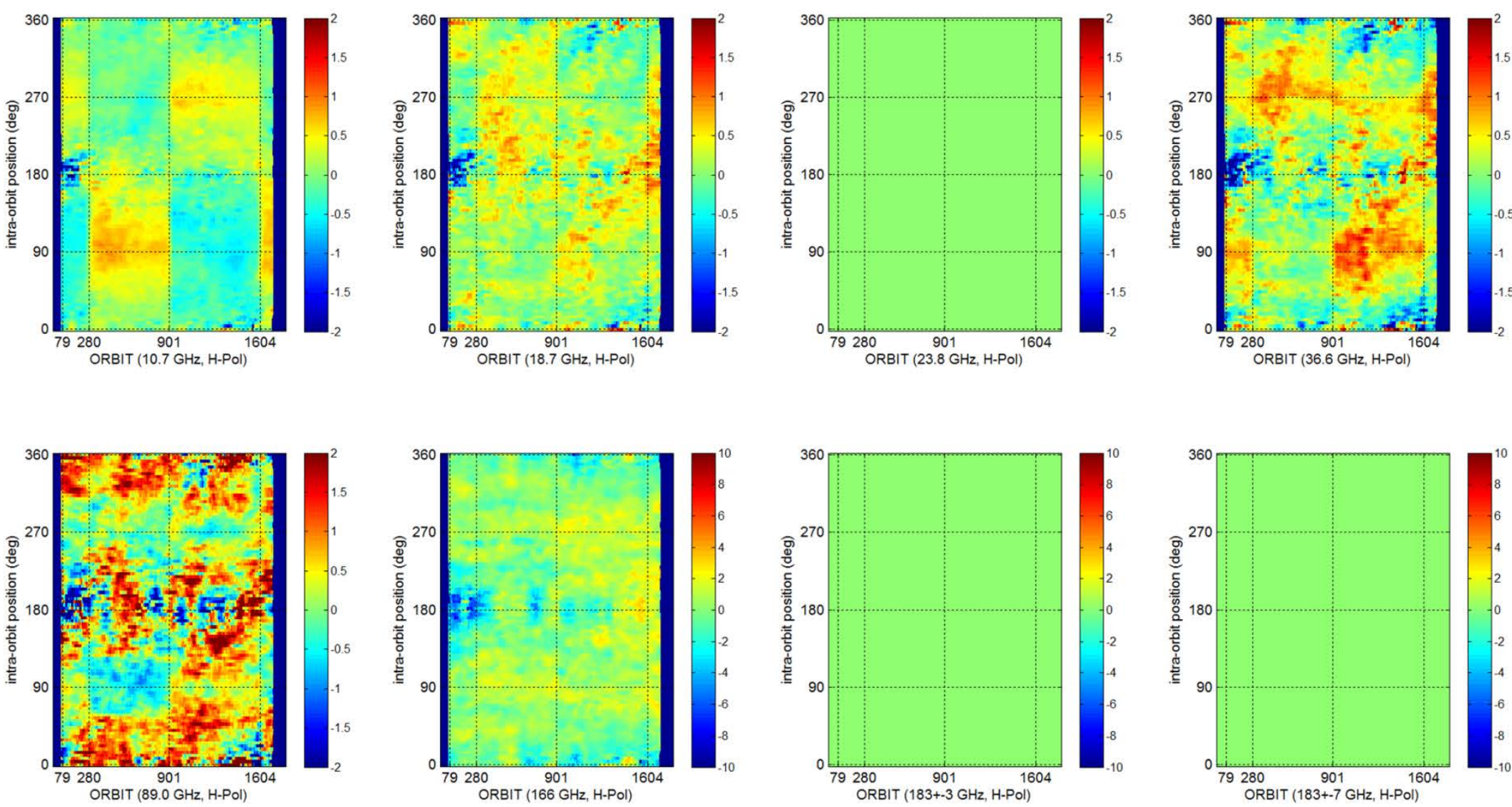


\section{GMI Minus RTM TA Difference Versus Orbit Number and Orbit Position: H-POL}

- Magnetic Correction Removes the orbital-dependent biases

- $23 \mathrm{GHz}$ and $183 \mathrm{GHz}$ do not have horizontal polarization channels
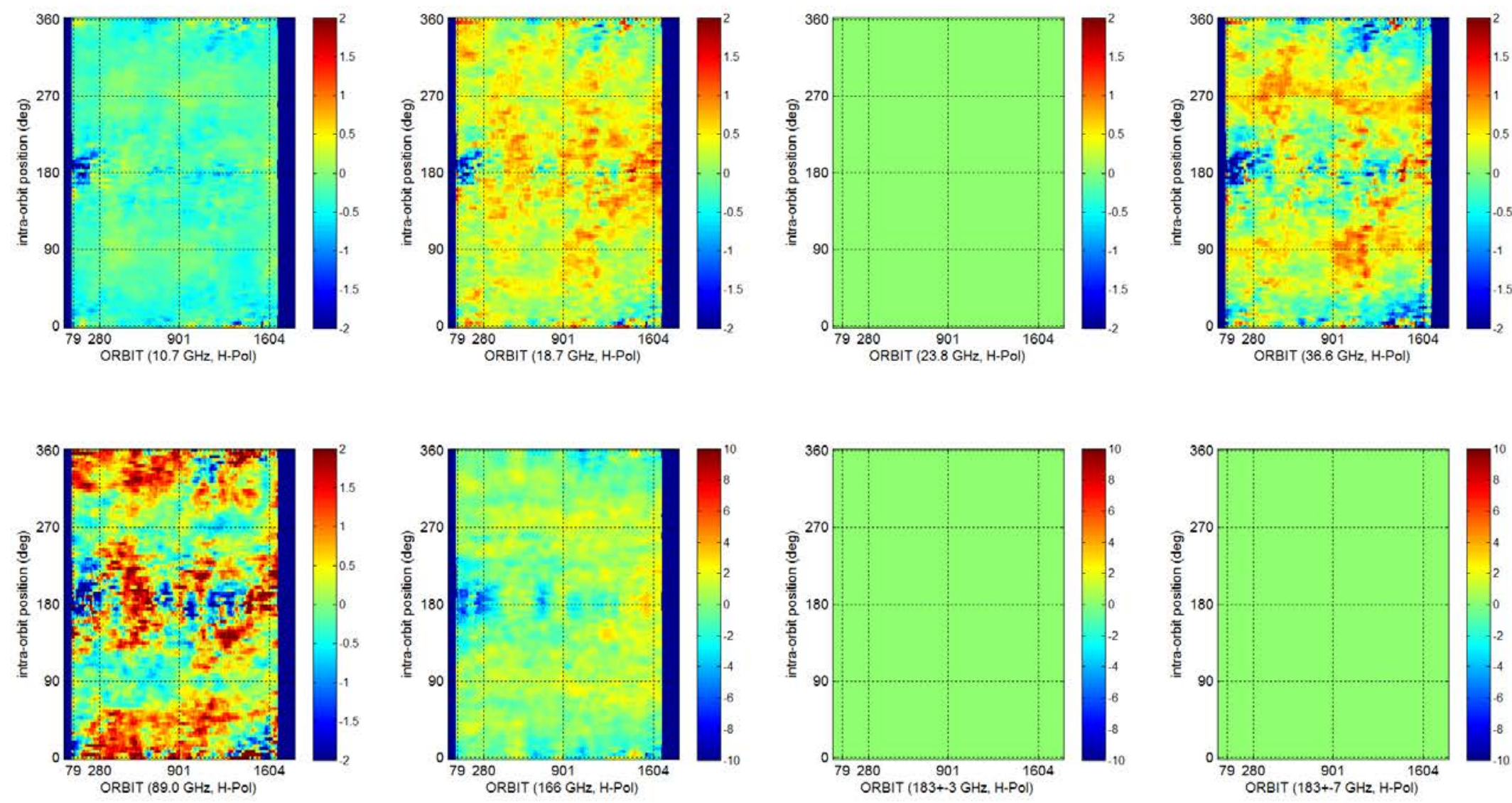


\section{GMI Minus RTM TA Difference Versus Orbit Number and Orbit Position: V-POL}

- Outstanding performance out of the box with magnetic correction
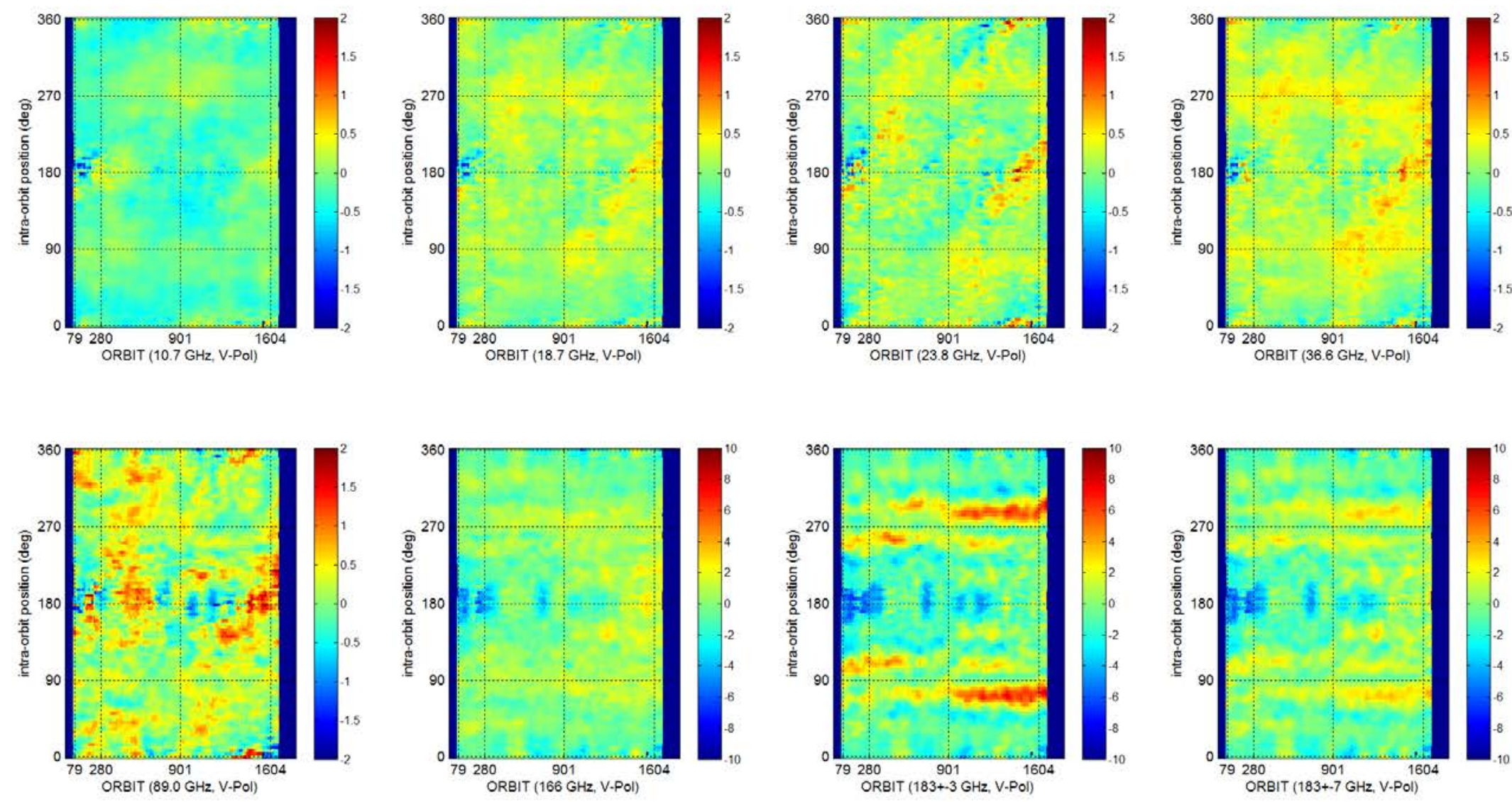


\section{GMI Minus RTM TA Difference Versus Solar Angles: V-POL}

- Analyzing the data this way allows for solar intrusions on the hot load to be identified

- The performance of the hot load is excellent
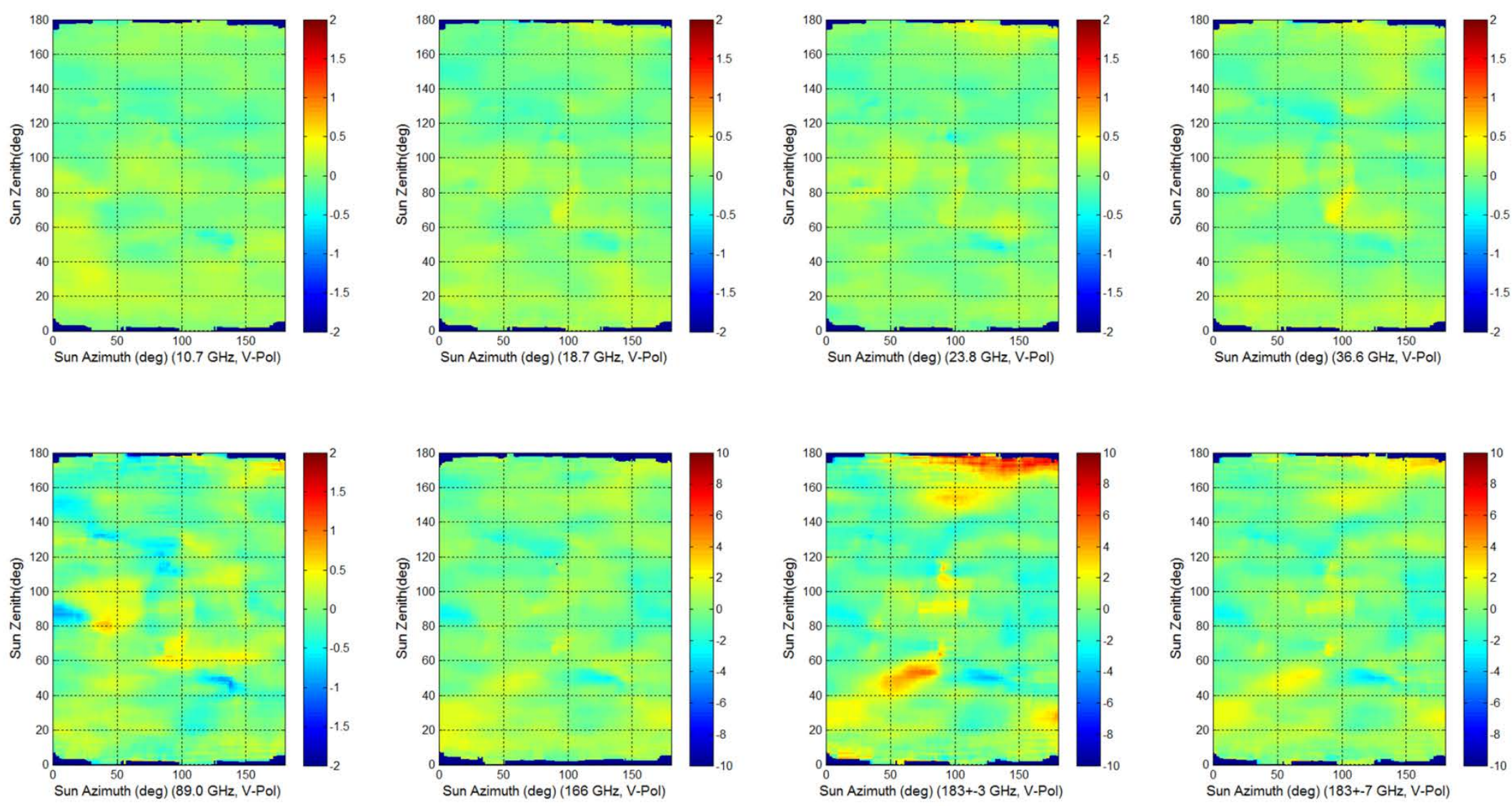


\section{Instrument-Induced Magnetic Correction}

$10 \mathrm{~V}$
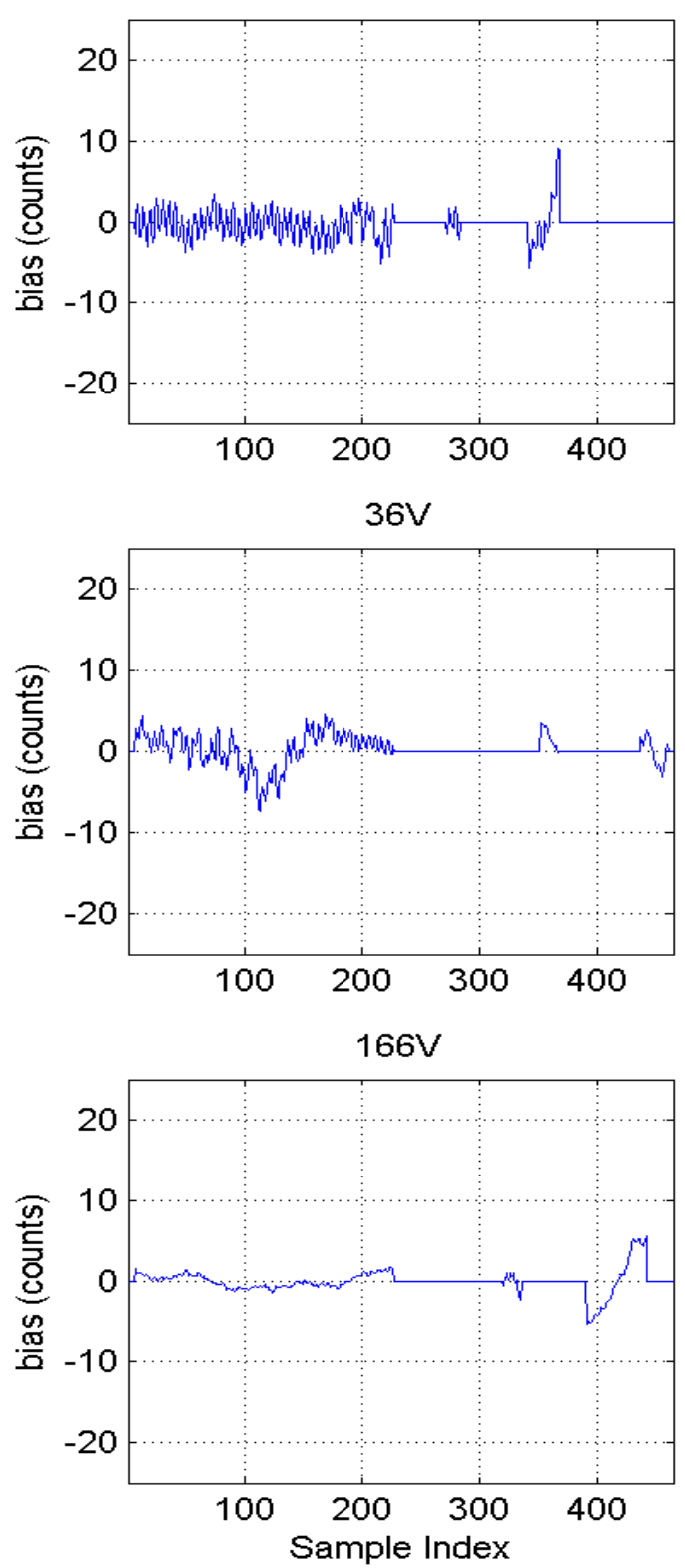

$10 \mathrm{H}$

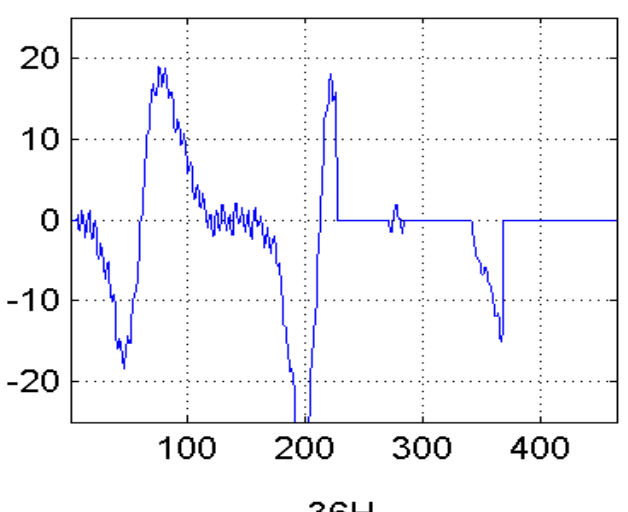

$36 \mathrm{H}$

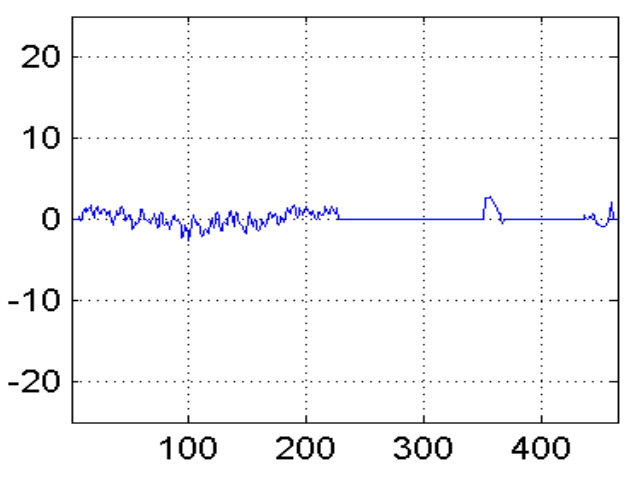

$166 \mathrm{H}$

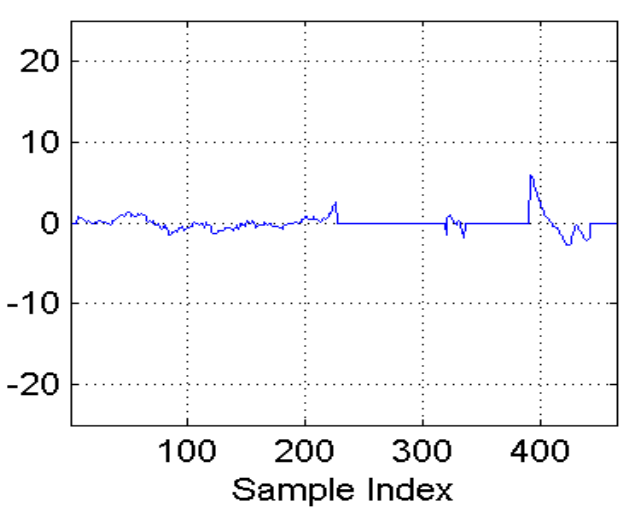

$18 \mathrm{~V}$ blue / 23V red

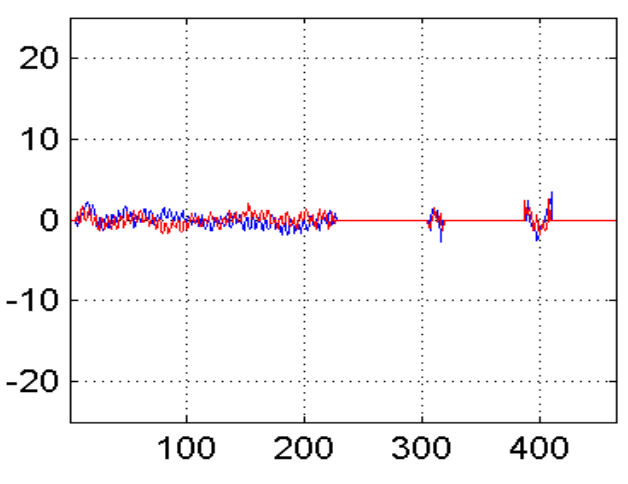

$89 \mathrm{~V}$

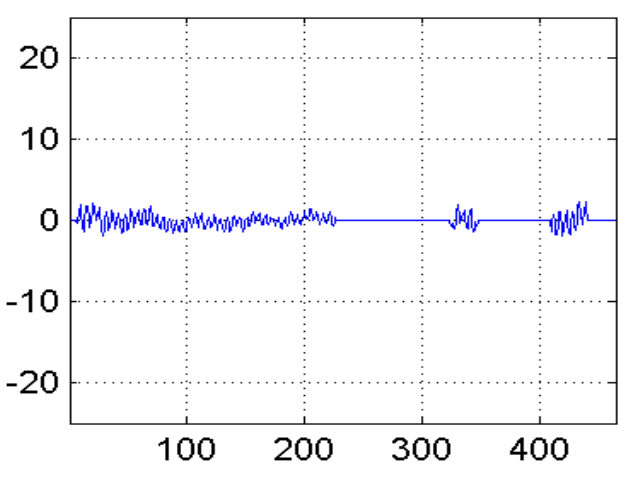

183VA

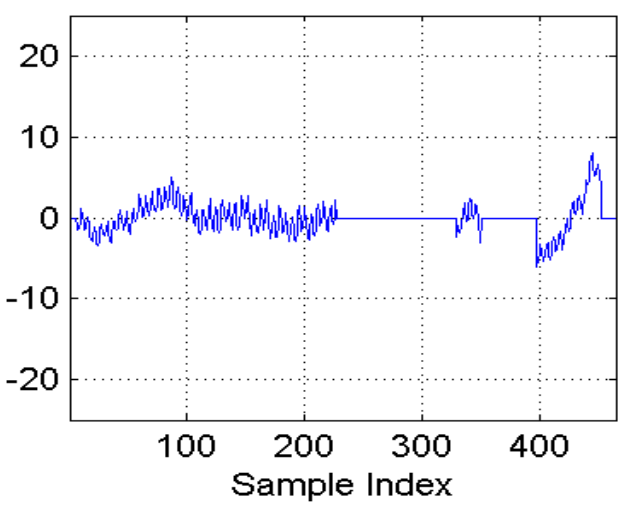

$18 \mathrm{H}$

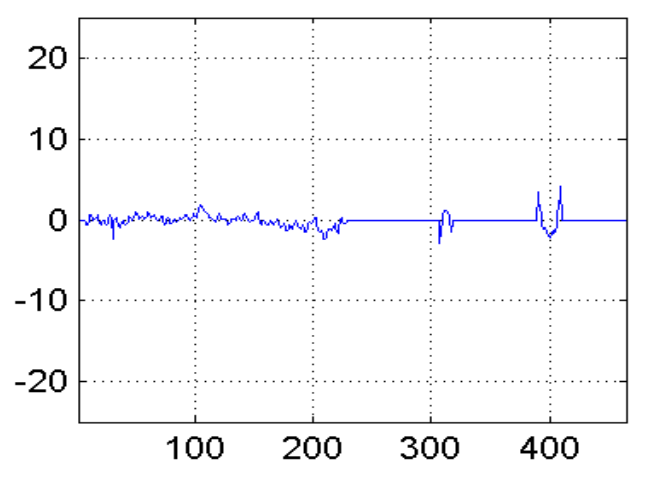

$89 \mathrm{H}$

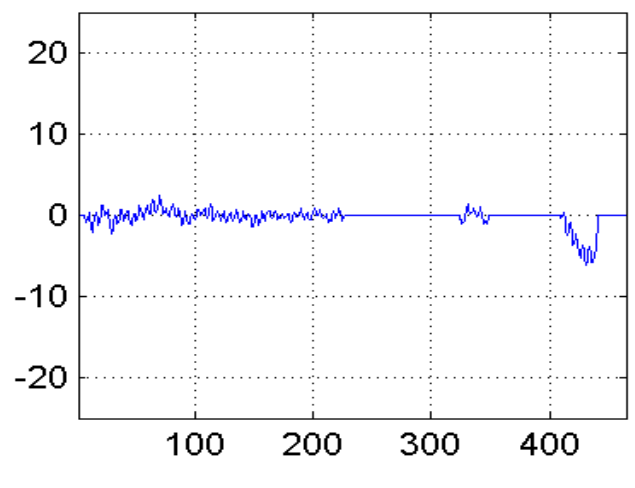

183VB

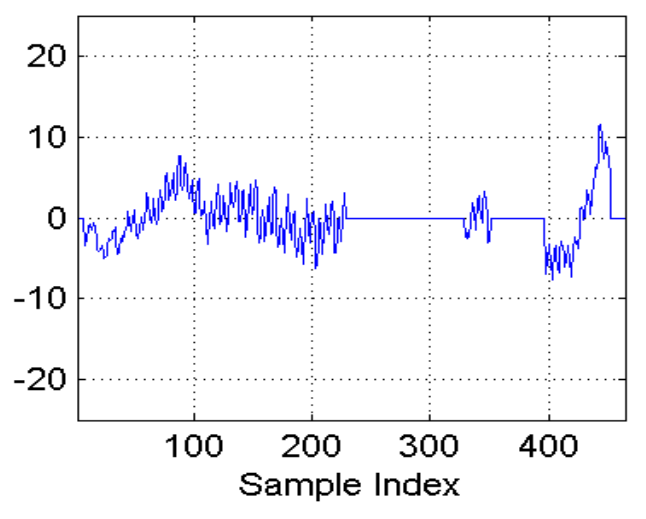




\section{GMI Summary}

- The initial commissioning of the GMI instrument is complete

- The first 3 months of data show that the instrument is performing well and meeting all requirements

- The NEDT and calibration uncertainty performance is much better than the requirements

- We are looking forward to many years of excellent data from GMI

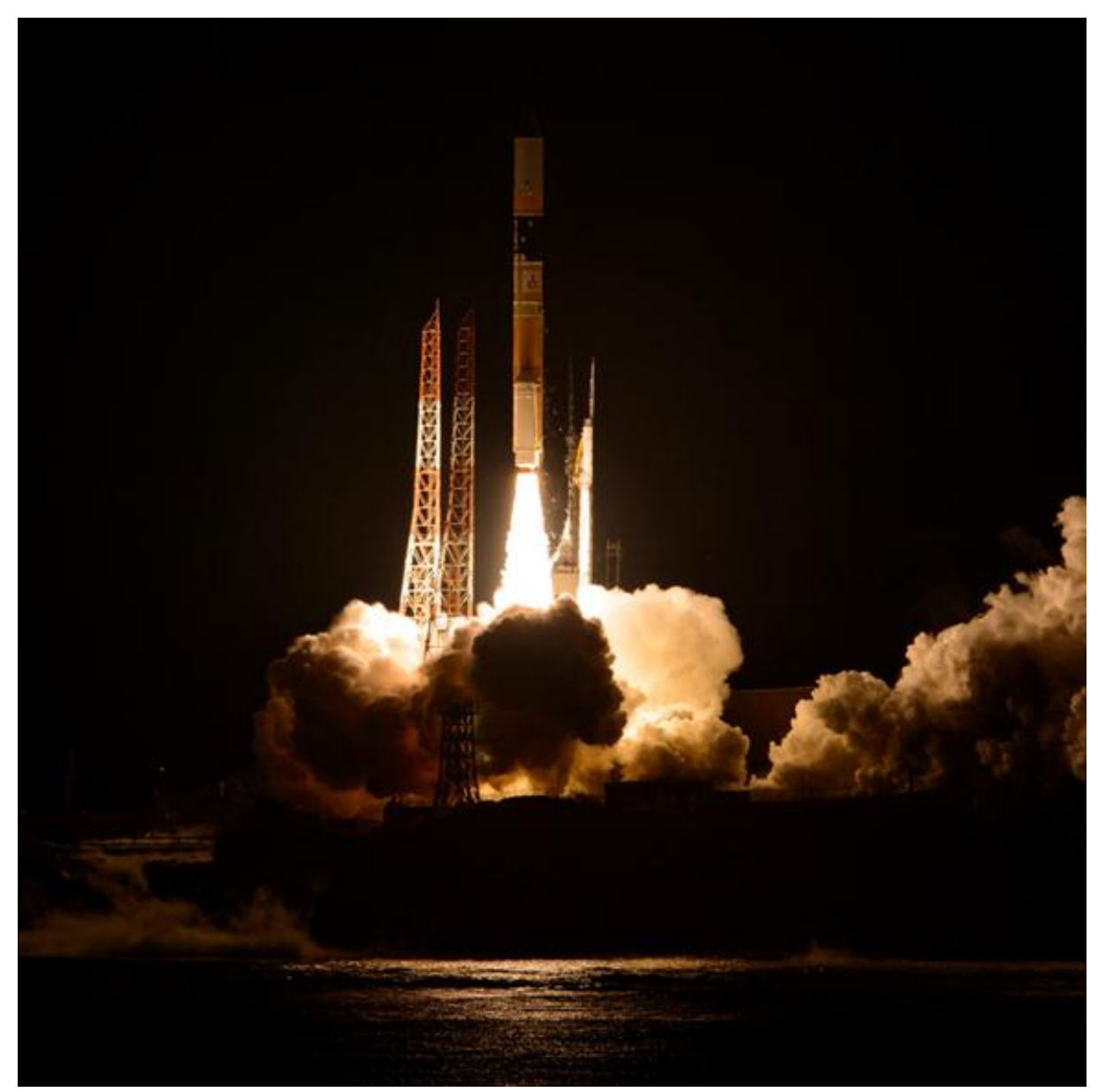

